\title{
Fecal microbiota of three bactrian camels (Camelus ferus and Camelus bactrianus) in China by high throughput sequencing of the V3-V4 region of the 16S rRNA gene
}

\author{
YUAN Lei ${ }^{1}$, QI Aladaer ${ }^{2,3 *}$, CHENG Yun ${ }^{1}$, SAGEN Guli ${ }^{1}$, QU Yuan ${ }^{4}$, LIU Bin $^{3}$ \\ ${ }^{1}$ Xinjiang Lop Nor Wild Camel National Nature Reserve, Urumqi 830011, China; \\ ${ }^{2}$ Animal Science Department, Xinjiang Agricultural University, Urumqi 830052, China; \\ ${ }^{3}$ Key Laboratory of Biogeography and Bioresources in Arid Land, Xinjiang Institute of Ecology and Geography, Chinese \\ Academy of Sciences, Urumqi 830011, China; \\ ${ }^{4}$ The People's Hospital of Xinjiang Uygur Autonomous Region, Urumqi 830001, China
}

\begin{abstract}
This study aimed to reveal the microbial diversity in the fecal samples of bactrian camels using the 16S rRNA sequencing analysis on the Illumina MiSeq platform. Three fecal samples were collected from two geographical regions in China. Operational taxonomic unit (OTU) clustering was performed by identifying an OTU at $97 \%$ sequence identity. The alpha and beta diversities were applied to estimate the differences in microbial diversity among the three fecal samples. Totally, 4409, 3151 and 4075 OTUs in the fecal samples were identified in the Lop Nor wild camel (Camelus ferus), the domestic camel (C. bactrianus) and Dunhuang wild camel (C. ferus), respectively. The majority of bactreria were affiliated with phylum Firmicutes and Bacteroidetes in the three samples. The wild camels had higher gastrointestinal tract microbial diversity than the domestic one, while the microbial composition of the Lop Nor wild camel shared higher similarity with domestic camel at the genus and family levels than that of the Dunhuang wild camel did. Our results may provide a theoretical basis for assessing their health conditions and may thus be useful for protecting the critically endangered species of $C$. ferus.
\end{abstract}

Keywords: bactrian camels; Camelus ferus; fecal microbiota; 16S rRNA; high-throughput sequencing

Citation: YUAN Lei, QI Aladaer, CHENG Yun, SAGEN Guli, QU Yuan, LIU Bin. 2017. Fecal microbiota of three bactrian camels (Camelus ferus and Camelus bactrianus) in China by high throughput sequencing of the V3-V4 region of the 16S rRNA gene. Journal of Arid Land, 9(1): 153-159. doi: 10.1007/s40333-016-0026-7

\section{Introdution}

Bactrian camels are characterized by two humps on their back. They belong to the family Camelidae, Tylopoda (suborder), and Artiodactyla (order). Bactrian camels are well-known for their tolerance to extreme temperature and drought conditions and they were domesticated very early. There are two strains of bactrian camels, one is so called wild bactrian camels, and the other is domestic ones. Domestic bactrian camels presently distribute in the wide range of Central Asia

\footnotetext{
*Corresponding author: QI Aladaer (E-mail: aqicas@foxmail.com)

The first and second authors contributed equally to this article.

Received 2016-03-25; revised 2016-11-19; accepted 2016-11-21

(C) Xinjiang Institute of Ecology and Geography, Chinese Academy of Sciences, Science Press and Springer-Verlag Berlin Heidelberg 2017
} 
including China and Mongolia and the local herders keep them basically for transportation purposes. Scientists have been debating on whether the two types of bactrian camels belong to the same species or not. For instance, Ji et al. (2009) explored the origin of domestic bactrian camels and the evolutionary relationship with the extant wild camels. They reported that bactrian camels appeared monophyletic in evolutionary origin and that the domestic bactrian camels might have originated from a single branch of wild populations. On the contrary, other researchers believed in the proposition that the wild bactrian camels were derived from the domestic ones that once returned to the wild and they preferred to using Camelus bactrianus as the scientific name of the two types (domestic and wild). Nevertheless, the International Union for Conservation of Nature (IUCN) insists on using C. bactrianus for the domestic ones and C. ferus for the wild ones. The wild bactrian camels (C. ferus) have been seriously threatened by human activities and also by crossbreeding with domestic camels (Hare, 2004). Indeed, a rather limited number of C. ferus have been found only in the Lop Nor of Xinjiang Uygur Autonomous Region of China, the Hexi Corridor of Gansu Province of China, and the Gobi part of Mongolia. Therefore, the IUCN has enrolled the wild bactrian camels (C. ferus) on the Red List Critically Endangered Species (i.e., CR level) in 2002. The Red List itself sufficiently illustrates the importance of the attempts to elevate the chances of their survivals (Yuan et al., 2015).

For studying rumen microbes, $16 \mathrm{~S}$ ribosomal RNA sequencing has become a mature technique widely used in studying beef cattle (Wright et al., 2007), dairy cattle (Whitford et al., 2001; King et al., 2011), and sheep (Wright et al., 2006, 2008). But, no studies have been conducted on the gastrointestinal microbial flora of bactrian camels (C. ferus and C. bactrianus). In this investigation, we studied and compared the intestinal microbial flora in the fecal samples of three bactrian camels from different origins by employing 16S rRNA gene high-throughput sequencing with a hope to provide a theoretical basis for assessing their health conditions and feeding structure changes and may thus be useful for protecting the critically endangered species of $C$. ferus.

\section{Materials and methods}

\subsection{Camel fecal samples}

Fecal samples of bactrian camels were collected from three healthy camels. The first one is an adult wild camel (YLT) living in Urumqi Wildlife Park that was originally from Lop Nor of Xinjiang Uygur Autonomous Region of China; the second one is an adult domestic camel (JLT) also living in Urumqi Wildlife Park; and the third one is a young wild camel (DHYLT) living in Dunhuang desert of Gansu Province of China. All fecal samples were collected freshly and frozen immediately into liquid nitrogen.

\subsection{DNA extraction, 16S rRNA gene PCR and sequencing}

DNA was extracted from fecal samples using TIANamp Stool DNA Kit (Tiangen Biotech. Co., Ltd., Beijing, China) in Xinjiang Institute of Ecology and Geography (CAS). DNA quantification was performed using the Qubit2.0 DNA Assay Kit (Sangon Biotech Co., Ltd, Shanghai, China). The primers 341f (CCTACGGGNGGCWGCAG) and 805R (GACTACHVGGGTATCTAATCC) were used for PCR amplification of the V3-V4 region of the 16S rRNA gene (Mizrahi-Man et al., 2013). PCR amplifications were performed in eppendorf mastercycler in a final volume of $50 \mu \mathrm{L}$ containing $10 \mathrm{ng}$ of genomic DNA, $0.5 \mu \mathrm{L}$ dNTP $(10 \mathrm{mM}$ each $), 0.5 \mu \mathrm{L}$ of each PCR primer $(50 \mathrm{uM})$ and $0.5 \mu \mathrm{L}$ of Taq $(5 \mathrm{U} / \mathrm{uL})$. Amplification was done using the following protocol: one denaturation step of $94^{\circ} \mathrm{C}$ for $3 \mathrm{~min} ; 5$ cycles of denaturation at $94^{\circ} \mathrm{C}$ for $15 \mathrm{~s}$ and extension at $65^{\circ} \mathrm{C}$ for $60 \mathrm{~s}, 20$ cycles of denaturation at $94^{\circ} \mathrm{C}$ for $1 \mathrm{~min}$, annealing at $55^{\circ} \mathrm{C}$ for $20 \mathrm{~s}$ and extension at $72^{\circ} \mathrm{C}$ for $30 \mathrm{~s}$, and a final extension at $72^{\circ} \mathrm{C}$ for $5 \mathrm{~min}$. Another $50 \mu \mathrm{L}$ reaction mixture contained $20 \mathrm{ng}$ of DNA, $0.5 \mu \mathrm{L}$ of dNTP $(10 \mathrm{mM}$ each), $0.5 \mu \mathrm{L}$ of each primer $(50 \mathrm{uM})$ and $0.5 \mu \mathrm{L}$ of Taq $(5 \mathrm{U} / \mu \mathrm{L})$ was used for PCR under the following conditions: denaturation at $95^{\circ} \mathrm{C}$ for $30 \mathrm{~s}, 5$ cycles of denaturation at $95^{\circ} \mathrm{C}$ for $15 \mathrm{~s}$, annealing at $55^{\circ} \mathrm{C}$ for $15 \mathrm{~s}$ and extension at $72^{\circ} \mathrm{C}$ for $30 \mathrm{~s}$, and a final extension at $72^{\circ} \mathrm{C}$ for $5 \mathrm{~min}$.

Amplification products were analyzed in agarose gel (1\%) using DNA ladder (100 bp) (Promega) as reference, and then recycled using DNA Recycle Kit, SK8131 (Sangon Biotech Co., Ltd, 
Shanghai, China), and finally quantified using Qubit2.0 DNA Assay Kit (Sangon Biotech Co., Ltd, Shanghai, China). A paired-end sequencing was performed using Illumina MiSeq platform (Illumina, San Diego, CA, USA).

\subsubsection{S rRNA sequence analysis}

First, the paired end reads were merged using FLASH v1.2.7. Each sample was separated based on its barcode sequence using a self-written script. Next, the reads of each sample were filtered by removing primer sequences, short sequences, and low-quality sequences using Prinseq software (PRINSEQ-lite 0.19.5, http://sourceforge.net/projects/prinseq/files/) and contaminating sequences from mitochondrial and chloroplast were filtered out according to the methods from $\mathrm{Wu}$ et al. (2016). Chimeric sequences were explored using Uchime software (http://www.mothur.org /wiki/Pre.cluster) with reference to Silva Small Subunit rRNA Database (SSU) and then removed. Finally, Uclust v1.1.579 (http://www.drive5.com/uclust/downloads11579.html) was used to align sequences with the SILVA 16S rRNA reference database (http://www.arb-silva.de/). An operational taxonomic unit (OTU) was identified taking 97\% sequence identity criterion as cut-off. Taxonomic classification of the resulting OTUs was performed using the Ribosomal Database Project (RDP) classifier by Naïve Bayesian assignment algorithm. Taxonomic assignment from phylum to genus level was realized at an $80 \%$ similarity threshold.

2.2.2 Microbial diversity and abundance analysis

To investigate the microbial diversity of each sample, we estimated the standard diversity and richness indices, including the Ace index (Chao and Shen, 2004), Chao1 index (Chao, 1984) and Shannon index (Chao and Shen, 2003) using Mothur software (Schloss et al., 2009). To estimate the microbial diversity differences between the three fecal samples, we performed beta diversity analysis by calculating the UniFrac distance between samples using principle component analysis (PCA, Hamady et al., 2010).

\section{Results}

\subsection{S rRNA sequences}

Most of the filtered reads were within 400-500 bp in length, averagely longer than $400 \mathrm{bp}$. Totally, 23,523-26,800 and 29,411 filtered sequences were obtained from two wild camels from Lop Nor and Dunhuang and one domestic camel, respectively. According to sequence alignment at a sequence identity cut-off value of $97 \%, 4409,4075$, and 3151 OTUs were identified correspondingly (Table 1).

According to the alpha diversity analysis, the Shannon values indicating the microbial diversity in JLT, YLT and DHYLT were 6.53, 6.98 and 6.82, respectively, demonstrating a good microbial diversity in all of the threes samples. The values of Shannon, Ace, and Chaol indices were all higher in the two wild camel samples than in the domestic one (Table 1).

\subsection{Microbial composition at different taxonomic levels}

Our following discussions start with the phylum level and end at the genus level (Fig. 1). At the phylum level, the proportion of Firmicutes was high in the identified microorganisms, with $53.59 \%$ in Lop Nor wild camel, $51.03 \%$ in domestic camel, and $75.41 \%$ in Dunhuang wild camel, followed by Bacteroidetes (correspondingly $30.77 \%, 32.31 \%$, and $13.74 \%$ in the three samples)

Table 1 Numbers of operational taxonomic units and microbial diversity indices in the three fecal samples of wild and domestic camels

\begin{tabular}{ccccccc}
\hline Sample_ID & Sequence & OTU & Shannon index & Ace index & Chao1 index & Coverage \\
\hline JLT & 29,411 & 3151 & 6.531 & 8285 & 6374 & 0.947 \\
YLT & 23,523 & 4409 & 6.976 & 18,588 & 12,314 & 0.885 \\
DHYLT & 26,800 & 4075 & 6.817 & 16,696 & 10,401 & 0.910
\end{tabular}

Notes: JLT: fecal sample from a domestic camel living in Urumqi Wildlife Park; YLT: fecal sample from a wild camel originally from Lop Nor also living in Urumqi Wildlife Park; DHYLT: fecal sample from a wild camel living in Dunhuang area. OUT: operational taxonomic unit. 
(a)
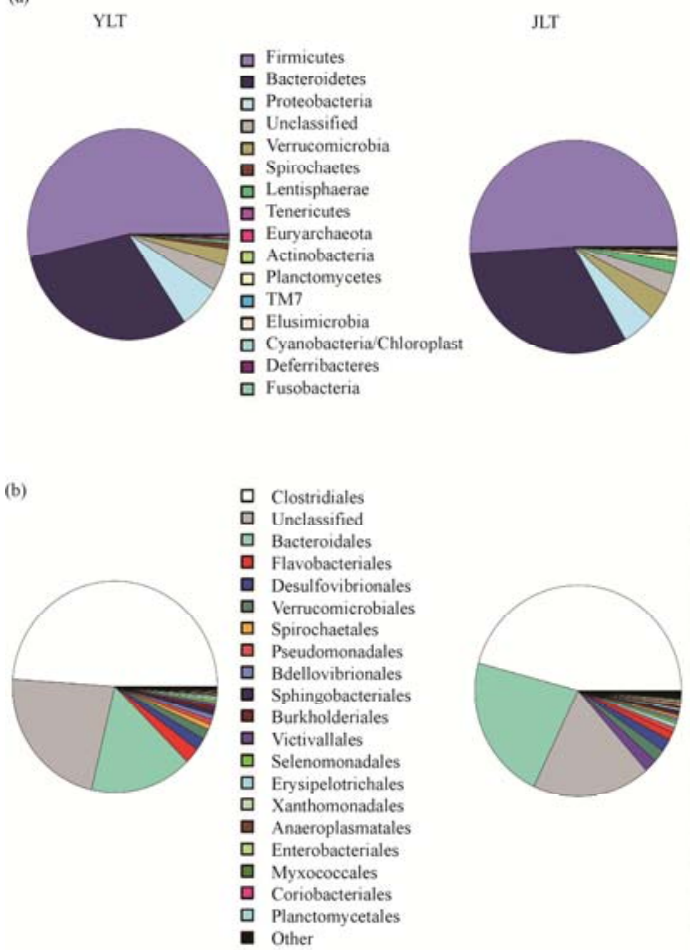

ㅁ Firmicutes

- Bacteroidetes

Verrucomicrobi.

ㄴ Unclassified

Lentisphaerae

Planctomycetes

- Spirochaetes

- Actinobacteria

Cyanobacteria/Chloroplast

- Tenericutes

Chloroflexi

Euryarchaeota

ㅁ TM7

ㅁ. Nitrospira

ㅁ Fibrobacteres

ㄷusobacteria

Deferribacteres

므 Synergistetes

- Other

므 Clostridiales

Bacteroidales

Unclassified

- Victivallales

․ Verrucomicrobiales

Desulfovibrionales

Flavobacteriales

Pseudomonadales

․ Planctomycetales

ㅁ. Selenomonadales

ㅁ Spirochaetales

- Bacillales

- Sphingobacteriales

E Eysipelotrichales

a Burkholderiales

ㅁ Enterobacteriales

․ Coriobacteriales

X Xanthomonadales

Myxococcales

- Other

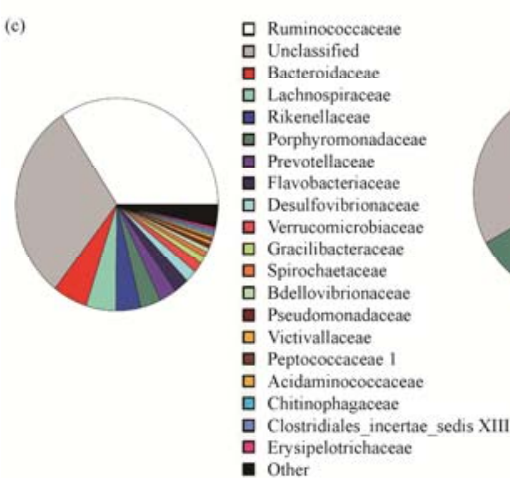

ㅁ Ruminococcaceae

ㅁ Unclassified

ㅁ Porphyromonadaceae

- Prevotellaceae

- Bacteroidaceae

- Rikenellaceae

ㅁ Lachnospiracea

ㅁ Victivallaceae

ㅁ Verrucomicrobiaceac

믈 Desulfovibrionaceae

- Flavobacteriaceac

- Planctomycetaceac

ㅁ Gracilibacteraceae

Clostridiales incertae sedis XIII

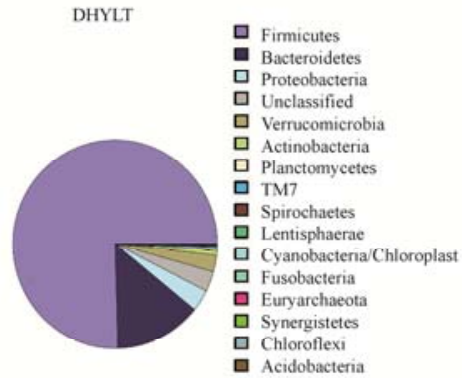

ㅁ Firmicutes

Bacteroidetes

$\square$ Unclassified

Verrucomicrobis

도으obacteria

ㅁ Lentisphaera

ㅁ Cyanobacteria/Chloroplast

ㅁ Fusobacteria

믈 Euryarchaeot:

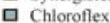

Acidobacteria

- Pseudomonadaceae

- Pseudomonadace

ㅁ. Peptococcaceae

ㅁ Acidaminococcacea

ㅁ Spirochaetaceae

ㅁ Erysipelotrichaceae

- Other

- Clostridiales

ㅁ Unclassified

ㅁ Bacteroidales

a Verrucomicrobiales

- Flavobacteriales

- Pseudomonadales

ㅁ Erysipelotrichales

- Desulfovibrionales

口 Enterobacteriales

- Coriobacteriales

- Sphingobacteriales

- Sphingobacteriales

ㅁ. Selenomonadales

- Burkholderiales

口 Bdellovibrionales

- Lactobacillales

口 Aeromonadales

- Victivallales

Spirochaetales

․ Rhizobiales

E. Other

Other

Unelosified

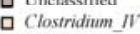

- Sponobacter

口 Bacteroides

Alistipes

- Parapnevotella

口 Paludibacter

O Oscillibacter

$\square$ Perrimonas

- Flavonifractor

- Acetivibrio

․ Subdivisions genera inceriae sedis

ㅁ Ruminococcaceae

ㅁ Unclassified

ㅁ Lachnospiraceae

․ Peptostreptococcaceae

Verrucomicrobiaceae

a Porphyromonadaceae

- Bacteroidaceae

- Clostridiales incertae sedis XIII

- Rikenellaceac

- Prevotellaceae

- Clostridiaceae 1

ㅁ Marinilabiaceae

- Flavobacteriaceae

ㅁ. Eubacteriaceae

G Gracilibacteraceae

- Pseudomonadaceae

- Erysipelotrichaceae

$\square$ Desulfovibrionacea

ㅁ Enterobacteriaceac

ㅁ Coriobacteriaceae

- Other

ㅁ Unclassified

ㅁ Clostridium_/I

ㅁ Spombacter

ㅁ Oscillibacter

Acetivibrio

- Clostridium XI

ㅁ Ruminococcus

Flavonifractor

Lachnospiracea incertae sedis

Clastridimom XIV

ㅁ Akkermansia

- Bacteroides

- Alistipes

- Papillibacier

口 Desulfovibrio

- Papillibacter

- Clostridium XIIT

Lachospiracea incertae sedis

- Psendoflavonifractor

Ornithobacterium

- Other

a Petadoflavonifractor

- Mogibacterium

ㅁ Clostridium sensu stricto

ㅁ. Anaerotruncus

- Canapnevolella

- Otostr

Fig. 1 Microbial composition in the three camels. The composition was classified at phylum (a), order (b), family (c), and genus (d) taxonomic levels. JLT: fecal sample from a domestic camel living in Urumqi Wildlife Park; YLT: fecal sample from a wild camel originally from Lop Nor also living in Urumqi Wildlife Park; DHYLT: fecal sample from a wild camel living in Dunhuang area. 
(Fig. 1a). At the phylum level, the similarity in the microbial composition was rather high among the three samples.

At the order level, Clostridiales was the predominant taxon in the three camels (accounting for $48.88 \%$ in Lop Nor wild camel, $45.79 \%$ in domestic camel and $68.9 \%$ in Dunhuang wild camel), followed by the order Bacteroidales. Victivallales, Verrucomicrobiales, and Desulfovibrionales bacteria had higher proportions in domestic camel, while Flavobacteriales, Desulfovibrionales and Verrucomicrobiales had higher proportions in Lop Nor wild camel, and the proportion of Verrucomicrobiales bacteria was relatively high in Dunhuang wild camel (Fig. 1b).

At the family level, the proportion of unidentified genera was almost same among the three samples. Ruminococcaceae was the largest taxon among the identified microbes in each sample, followed by Porphyromonadaceae in the domestic camel, by Bacteroidaceae in the Lop Nor wild camel, and by Lachnospiraceae in the Dunhuang wild camel. Additionally, bacteria of Lachnospiraceae, Rikenellaceae and Porphyromonadaceae had relatively large proportions in the Lop Nor wild camel; bacteria of Porphyromonadaceae, Prevotellaceae, Bacteroidaceae, Rikenellaceae and Lachnospiraceae accounted for relatively large proportions in the domestic camel. In the Dunhuang wild camel, the bacteria of Peptostreptococcaceae had a larger proportion (Fig. 1c). The microbial composition at the family level in the Lop Nor wild camel was quite similar to that in the domestic camels but rather different from that in the Dunhuang wild camel.

At the genus level, the unidentified genera had similar proportions in the total identified microbial communities among the three camels. Clostridium IV, belonging to the order Clostridiales under the family Ruminococcaceae of the phylum Firmicutes, was the largest group in all the three samples, accounting for $7.03 \%$ in the Lop Nor wild camel, $7.86 \%$ in the domestic camel and $8.36 \%$ in the Dunhuang wild camel, followed by Sporobacter as the second largest genus in each one of the samples. In addition, the Lop Nor wild camel also had abundant bacteria belonging to Sporobacter, Bacteroides, Oscillibacter, Alistipes and Flavonifractor, each with a proportion of $3.79 \%$ or higher. The domestic camel contained abundant bacteria from the genus Sporobacter, Bacteroides, Alistipes, Paraprevotella, Paludibacter, and Oscillibacter, each with a proportion of $3.54 \%$ or higher. The Dunhuang wild camel contained various microbes in Sporobacter, Oscillibacter, Acetivibrio, Clostridium XI, each with a proportion of $3.62 \%$ or higher (Fig. 1d). Overall, the genera constituting the microbial populations in the three samples are almost the same and the general composition of Lop Nor wild camel is similar to that of the domestic camel and different from Dunhuang wild camel. Again, the clustering analysis of the genetic distance data shows that the domestic camel and Lop Nor wild camel had a higher degree of similarity (Fig. 2a), being consistent with the result of principle component analysis (PCA) analysis (Fig. 2b).

(a)

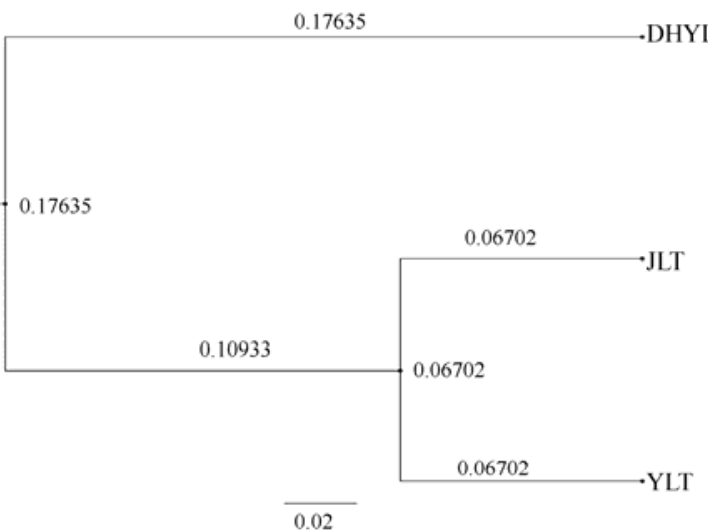

(b)

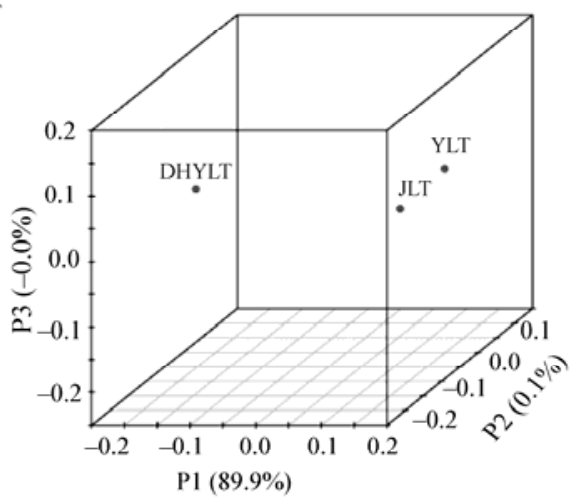

Fig. 2 Clustering analysis (weighted) by the genetic distance of the three camels (a); 3-D spatial plots (weighted) from distance principle component analysis of the three camels (b). JLT: fecal sample from a domestic camel living in Urumqi Wildlife Park; YLT: fecal sample from a wild camel originally from Lop Nor also living in Urumqi Wildlife Park; DHYLT: fecal sample from a wild camel living in Dunhuang area. 


\section{Discussion and conclusions}

In this study, fecal microbial composition and the diversity at different taxonomic units of two wild bactrian camels and one domestic bactrian camel were investigated using the 16S rRNA sequencing. The results indicated that both the wild camels and the domestic camel had abundant microbial species in their fecal samples. Firmicutes and Bacteroidetes were predominant phyla in the three camels, being quite consistent with the results reported by Samsudin et al. (2011). The documented predominancies of Firmicutes and Bacteroidetes in the bactrian camels are quite similar to the reported predominancies of Firmicutes and Bacteroidetes in other herbivorous mammals such as horses (Zhao et al., 2016). This study shows that Clostridium IV bacteria, belonging to Clostridia which is one of the three classes of the phylum Firmicutes (Clostridia, Bacilli and Mollicutes; Wolf et al., 2004) was the most abundant microorganisms at the genus level with a proportion of higher than $7 \%$ in each of the three camels. It was reported by Bäckhed et al. (2004) that Firmicutes was associated with fatty acid absorption and the abundance of Firmicutes was observed to be proportional to the obesity levels in the mice (Bäckhed et al., 2004). Costa et al. (2012) showed that healthy horses were predominated by Firmicutes (68\%), however horses with colitis were predominated by Bacteroidetes $(40 \%)$. Therefore, Furmicutes is likely the main reason for keeping the camel healthy digestion system and efficiently converting foods to the energy and fat.

Overall, the microbial composition at the phylum level was similar among the three camels. However, the similarities at the genus and family levels were higher between the Lop Nor wild camel and the domestic camel than between the Lop Nor wild camel and the Dunhuang wild camel. This finding was further confirmed by the PCA and clustering analysis, implying that similar age stage or living environment could be the controlling factors of the microbial composition. Specifically, both the Lop Nor wild camel and the domestic camel were adult and housed in similar living environments. In contrast, the Dunhuang wild camel was at its weaning stage and feeding on natural vegetation. Additionally, alpha diversity analysis revealed that the wild camels had more abundant microbial flora than the domestic camel, and the lower abundant microbial flora in the domestic camel may be resulted from the fact that the domestic camel has well adapted to the diet of grazing in domestic environment for a long-time, leading to a reduced gastrointestinal microbial diversity.

In conclusion, Clostridium IV, Ruminococcaceae, Clostridiales and Firmicutes were the largest taxa at the genus, family, order and phylum levels in all the three bactrian camels. Wild camels had higher fecal microbial diversity than the domestic one. Domestic camel and Lop Nor wild camel shared a high degree of similarity in intestinal microbial composition. The results may be used for assessing their health conditions associated with their food intakes and may thus be useful for protecting the critically endangered species of $C$. ferus. Finally, we suggest that future studies should be directed to understanding the influences of geographical differences and health conditions to the microbial composition in the C. ferus.

\section{Acknowledgements}

This research was supported by the Xinjiang Lop Nur Wild Camels National Reserve Comprehensive Scientific Research Projects by The Environmental Protection Agency of China (20100228).

\section{References}

Bäckhed F, Ding H, Wang T, et al. 2004. The gut microbiota as an environmental factor that regulates fat storage. Proceedings of the National Academy Sciences of the United States of America, 101(44): 15718-15723.

Chao A. 1984. Nonparametric estimation of the number of classes in a population. Scandinavian Journal of Statistics, 11: 265-270.

Chao A, Shen T J. 2003. Nonparametric estimation of Shannon's index of diversity when there are unseen species in sample. Environmental and Ecological Statistics, 10(4): 429-443.

Chao A, Shen T J. 2004. Nonparametric prediction in species sampling. Journal of Agricultural, Biological, and Environmental 
Statistics, 9(3): 253-269.

Costa M C, Arroyo LG, Allen-Vercoe E, et al. 2012. Comparison of the fecal microbiota of healthy horses and horses with colitis by high throughput sequencing of the V3-V5 region of the 16S rRNA gene. PLoS ONE, 7(7): e41484.

Hamady M, Lozupone C, Knight R. 2010. Fast UniFrac: facilitating high-throughput phylogenetic analyses of microbial communities including analysis of pyrosequencing and PhyloChip data. The ISME Journal, 4(1): 17-27.

Hare J. 2004. The wild Bactrian camel; a critically endangered species. Endangered Species Update, 21(1): 32-35.

Ji R, Cui P, Ding F, et al. 2009. Monophyletic origin of domestic bactrian camel (Camelus bactrianus) and its evolutionary relationship with the extant wild camel (Camelus bactrianus ferus). Animal Genetics, 40(4): 377-382.

King E E, Smith R P, St-Pierre B, et al. 2011. Differences in the rumen methanogen populations of lactating Jersey and Holstein dairy cows under the same diet regimen. Applied and Environmental Microbiology, 77(16): 5682-5687.

Mizrahi-Man O, Davenport E R, Gilad Y. 2013. Taxonomic classification of bacterial 16S rRNA genes using short sequencing reads: evaluation of effective study designs. PLoS ONE, 8(1): e53608.

Samsudin A A, Evans P N, Wright A D G, et al. 2011. Molecular diversity of the foregut bacteria community in the dromedary camel (Camelus dromedarius). Environmental Microbiology, 13(11): 3024-3035.

Schloss P D, Westcott S L, Ryabin T, et al. 2009. Introducing mothur: open-source, platform-independent, community-supported software for describing and comparing microbial communities. Applied and Environmental Microbiology, 75(23): 7537-7541.

Whitford M F, Teather R M, Forster R J. 2001. Phylogenetic analysis of methanogens from the bovine rumen. BMC Microbiology, 1(1): 5.

Wolf M, Müller T, Dandekar T, et al. 2004. Phylogeny of Firmicutes with special reference to Mycoplasma (Mollicutes) as inferred from phosphoglycerate kinase amino acid sequence data. International Journal of Systematic and Evolutionary Microbiology, 54(3): 871-875.

Wright A D G, Toovey A F, Pimm C L. 2006. Molecular identification of methanogenic archaea from sheep in Queensland, Australia reveal more uncultured novel archaea. Anaerobe, 12(3): 134-139.

Wright A D G, Auckland C H, Lynn D H. 2007. Molecular diversity of methanogens in feedlot cattle from Ontario and Prince Edward Island, Canada. Applied and Environmental Microbiology, 73(13): 4206-4210.

Wright A D G, Ma X L, Obispo N E. 2008. Methanobrevibacter phylotypes are the dominant methanogens in sheep from Venezuela. Microbial Ecology, 56(2): 390-394.

Wu X Y, Zhang H H, Chen J, et al. 2016. Comparison of the fecal microbiota of dholes high-throughput Illumina sequencing of the V3-V4 region of the 16S rRNA gene. Applied Microbiology and Biotechnology, 100(8): 3577-3586.

Yuan L, Ma H, Cheng Y, et al. 2015. Qualitative study of wild camels (Camelus ferus) home range in Lop Nur, China. Biodiversity Science, 23(3): 314-320. (in Chinese)

Zhao Y P, Li B, Bai D Y, et al. 2016. Comparison of fecal microbiota of Mongolian and Thoroughbred Horses by high-throughput sequencing of the V4 Region of the 16S rRNA gene. Asian-Australasian Journal of Animal Sciences, 29(9): 1345-1352. 